PROCEEDINGS OF THE

AMERICAN MATHEMATICAL SOCIETY

Volume 137, Number 7, July 2009, Pages 2169-2174

S 0002-9939(09)09640-3

Article electronically published on February 12, 2009

\title{
REGULARITY INDEX OF HILBERT FUNCTIONS OF POWERS OF IDEALS
}

\author{
TRAN NAM TRUNG
}

(Communicated by Bernd Ulrich)

\begin{abstract}
Let $I$ be a homogeneous ideal of a Noetherian standard graded algebra $A$ over an Artinian ring $A_{0}$, and let $M$ be a finitely generated graded $A$-module. It is shown that the regularity index of the Hilbert function of $I^{n} M$ is a linear function of $n$ for all $n$ large enough.
\end{abstract}

\section{INTRODUCTION}

Throughout this paper we assume that $A$ is a Noetherian standard graded algebra over an Artinian ring $A_{0}$. For a finitely generated graded $A$-module $M, H_{M}(m):=$ $\ell_{A_{0}}\left(M_{m}\right), m \in \mathbb{Z}$, is called the Hilbert function of $M$. It is well-known that there is a polynomial $P_{M}(x) \in \mathbb{Q}[x]$ called the Hilbert polynomial of $M$ such that $H_{M}(m)=P_{M}(m)$ for all $m$ large enough. The regularity index of the Hilbert function of $M$ is defined by

$$
r i(M):=\min \left\{m_{0} \mid H_{M}(m)=P_{M}(m) \forall m \geqslant m_{0}\right\} .
$$

Let $I$ be a homogeneous ideal of $A$. In this paper, we are interested in the following problem posed in [3]: is $\operatorname{ri}\left(I^{n} M\right)$ a linear function of $n$ for all $n \gg 0$ ? This problem comes from the asymptotic behaviour of the so-called Castelnuovo-Mumford regularity $\operatorname{reg}\left(I^{n} M\right)$. It was first shown in 2 and 4 for the case $M=A$ being a polynomial ring over a field, and then in [6] for the general case that $\operatorname{reg}\left(I^{n} M\right)$ is a linear function of $n$ for all $n \gg 0$. Since the regularity index $r i\left(I^{n} M\right)$ is less than or equal to the Castelnuovo-Mumford regularity $\operatorname{reg}\left(I^{n} M\right)+1$, it is bounded by a linear function of $n$.

L. T. Hoa and E. Hyry showed that $r i\left(I^{n}\right)$ is a linear function of $n$ for all $n \gg 0$ if $I$ is a polynomial ideal generated in one or two degrees (see [3, Lemma 5 and Theorem 3]). The coefficient of this function is a generating degree of $I$. Their method is based on a bigraded free resolution of the Rees algebra $\mathfrak{R}(I)$ of $I$. To deal with the general case, they used the Hilbert-Poincaré series of $\mathfrak{R}(I)$ to translate the above problem to a purely combinatorial problem in polynomials of one variable (see [3]). Our method here is somewhat different from the suggestion by Hoa and Hyry. We also translate the above problem to a combinatorial problem. In general, instead of studying polynomials of one variable separately as in $\underline{3}$, we

Received by the editors October 29, 2007, and, in revised form, March 18, 2008.

2000 Mathematics Subject Classification. Primary 13D40.

Key words and phrases. Hilbert function, Hilbert polynomial, Hilbert-Poincaré series.

(C)2009 American Mathematical Society 
study them together in an interaction with a formal power series in two variables (see Theorem 11). By this method, we can prove the following main result.

Main Theorem. Let I be a homogeneous ideal of a standard graded algebra A over an Artinian ring $A_{0}$, and let $M$ be a $\mathbb{Z}$-graded A-module. Assume that $I^{n} M \neq 0$ for all $n \geqslant 1$. Then the regularity index $\operatorname{ri}\left(I^{n} M\right)$ is a linear function of $n$ for all $n$ large enough. The leading coefficient of this function is one of the generating degrees of $I$.

\section{A COMbinatorial Result}

In the paper we make the following convention: The degree of the zero polynomial is -1 .

Theorem 1. Given a sequence of polynomials $P_{0}(x), P_{1}(x), P_{2}(x), \ldots \in \mathbb{Q}[x]$, assume that there are a polynomial $P(x, y) \in \mathbb{Q}[x, y]$ and non-negative integers $\nu_{1}, \ldots, \nu_{p}, n_{1}, \ldots, n_{p}$ such that

$$
\sum_{n=0}^{\infty} P_{n}(x) y^{n}=\frac{P(x, y)}{\left(1-y x^{\nu_{1}}\right)^{n_{1}} \cdots\left(1-y x^{\nu_{p}}\right)^{n_{p}}} .
$$

Then $\operatorname{deg} P_{n}(x)$ is a linear function of $n$ for all $n \gg 0$. Moreover, if $P(x, y)$ is not divisible by $\left(1-y x^{\nu_{1}}\right)^{n_{1}} \cdots\left(1-y x^{\nu_{p}}\right)^{n_{p}}$, then the leading coefficient of this function is one of the numbers $\nu_{1}, \ldots, \nu_{p}$.

Proof. We may assume that $\nu_{1}<\nu_{2}<\cdots<\nu_{p}$ and $n_{i} \geqslant 1$ for all $i=1, \ldots, p$. For each $i=1, \ldots, p$, let

$$
Q_{x, i}:=\prod_{j \neq i}\left(1-y x^{\nu_{j}}\right)^{n_{j}} .
$$

In the polynomial ring $\mathbb{Q}(x)[y]$ of the variable $y$ over the field $\mathbb{Q}(x)$, each polynomial $1-y x^{\nu_{i}}$ is irreducible and any two polynomials $1-y x^{\nu_{i}}$ and $1-y x^{\nu_{j}}$ are coprime for $i \neq j$. Therefore, the polynomials $Q_{x, 1}(y), Q_{x, 2}(y), \ldots, Q_{x, p}(y)$ are coprime and we can find $p$ polynomials $A_{x, 1}(y), A_{x, 2}(y), \ldots, A_{x, p}(y) \in \mathbb{Q}(x)[y]$ such that

$$
A_{x, 1}(y) Q_{x, 1}(y)+A_{x, 2}(y) Q_{x, 2}(y)+\cdots+A_{x, p}(y) Q_{x, p}(y)=1 .
$$

This implies

$$
\frac{1}{\left(1-y x^{\nu_{1}}\right)^{n_{1}} \cdots\left(1-y^{\nu_{p}}\right)^{n_{p}}}=\sum_{i=1}^{p} \frac{A_{x, i}(y)}{\left(1-y x^{\nu_{i}}\right)^{n_{i}}}
$$

and

$$
\sum_{n=0}^{\infty} P_{n}(x) y^{n}=\frac{P(x, y)}{\left(1-y x^{\nu_{i}}\right)^{n_{i}} \cdots\left(1-y x^{\nu_{p}}\right)^{n_{p}}}=\sum_{i=1}^{p} \frac{P(x, y) A_{x, i}(y)}{\left(1-y x^{\nu_{i}}\right)^{n_{i}}} .
$$

Let $P(x, y) A_{x, i}(y)=a_{i 0}(x)+a_{i 1}(x) y+\cdots+a_{i m_{i}}(x) y^{m_{i}}$, where $a_{i 0}(x), \ldots, a_{i m_{i}}(x) \in$ $\mathbb{Q}(x)$. Fix $1 \leqslant i \leqslant p$. Since

$$
\frac{1}{\left(1-y x^{\nu_{i}}\right)^{n_{i}}}=\sum_{m=0}^{\infty}\left(\begin{array}{c}
m+n_{i}-1 \\
n_{i}-1
\end{array}\right) x^{m \nu_{i}} y^{m}
$$


we have

$$
\begin{aligned}
\frac{P(x, y) A_{x, i}(y)}{\left(1-y x^{\nu_{i}}\right)^{n_{i}}} & =\sum_{n=0}^{\infty}\left[\sum_{j=0}^{m_{i}}\left(\begin{array}{c}
n-j+n_{i}-1 \\
n_{i}-1
\end{array}\right) a_{i j}(x) x^{(n-j) \nu_{i}}\right] y^{n} \\
& =\sum_{n=0}^{\infty}\left[\sum_{j=0}^{m_{i}}\left(\begin{array}{c}
n-j+n_{i}-1 \\
n_{i}-1
\end{array}\right) a_{i j}(x) x^{-j \nu_{i}}\right] x^{n \nu_{i}} y^{n} \\
& =\sum_{n=0}^{\infty} R_{i}(x, n) x^{n \nu_{i}} y^{n},
\end{aligned}
$$

where

$$
R_{i}(x, n)=\sum_{j=0}^{m_{i}}\left(\begin{array}{c}
n-j+n_{i}-1 \\
n_{i}-1
\end{array}\right) a_{i j}(x) x^{-j \nu_{i}} .
$$

Hence, by (11), we get

$$
\sum_{n=0}^{\infty} P_{n}(x) y^{n}=\sum_{n=0}^{\infty}\left[\sum_{i=0}^{p} R_{i}(x, n) x^{n \nu_{i}}\right] y^{n} .
$$

This gives

$$
P_{n}(x)=\sum_{i=0}^{p} R_{i}(x, n) x^{n \nu_{i}}
$$

Let $n_{0}=\max \left\{m_{1}, \ldots, m_{p}\right\}$. For all $n \geqslant n_{0}$, the right side of (2) is the value of a polynomial $R_{x, i}(y) \in \mathbb{Q}(x)[y]$. This means that $R_{i}(x, n)=R_{x, i}(n)$ for all $n \geqslant n_{0}$. Choose $p$ polynomials $Q_{1}(x, y), \ldots, Q_{p}(x, y) \in \mathbb{Q}[x, y]$ and a polynomial $D(x) \in \mathbb{Q}[x]$ with $D(x) \neq 0$ such that

$$
R_{x, i}(y)=\frac{Q_{i}(x, y)}{D(x)} \text { for all } i=1, \ldots, p .
$$

By (3), we then get

$$
D(x) P_{n}(x)=\sum_{i=1}^{p} Q_{i}(x, n) x^{n \nu_{i}} \text { for all } n \geqslant n_{0} .
$$

There are two cases:

Case 1. $Q_{1}(x, y)=\cdots=Q_{p}(x, y)=0$. Then $P_{n}(x)=0$ for all $n \geqslant n_{0}$ and $\operatorname{deg} P_{n}(x)=-1$ for all $n \geqslant n_{0}$. This case is equivalent to the condition that $P(x, y)$ is divisible by $\left(1-y x^{\nu_{1}}\right)^{n_{1}} \cdots\left(1-y x^{\nu_{p}}\right)^{n_{p}}$.

Case 2. Not all $Q_{1}(x, y), \ldots, Q_{p}(x, y)$ are zero. Assume $Q_{t}(x, y) \neq 0$ for some $t=1, \ldots, p$. Let

$$
Q_{t}(x, y)=c_{0}(y)+c_{1}(y) x+\cdots+c_{d_{t}}(y) x^{d_{t}},
$$

where $d_{t}=\operatorname{deg}_{x}\left(Q_{t}(x, y)\right) \geqslant 0$ and $c_{0}(y), c_{1}(y), \ldots, c_{d_{t}}(y) \in \mathbb{Q}[y]$ with $c_{d_{t}}(y) \neq 0$. Since $c_{d_{t}}(y) \neq 0$, there is $\lambda_{t}$ such that $c_{d_{t}}(n) \neq 0$ for all $n \geqslant \lambda_{t}$. Therefore

$$
\operatorname{deg}\left(Q_{t}(x, n) x^{n \nu_{t}}\right)=n \nu_{t}+d_{t} \text { for all } n \geqslant \lambda_{t} .
$$


Let $k=\max \left\{i \mid Q_{i}(x, y) \neq 0\right\}$. If $Q_{t}(x, y) \neq 0$ and $t \neq k$, then $t<k$. Since $\nu_{t}<\nu_{k}$, by (5) we get

$$
\operatorname{deg}\left(Q_{t}(x) x^{\nu_{t} n}\right)<\operatorname{deg}\left(Q_{k}(x) x^{\nu_{k} n}\right) \text { for all } n>\max \left\{d_{t}-d_{k}+1, \lambda_{t}, \lambda_{k}\right\} .
$$

Let $N=\max \left\{n_{0}, d_{t}-d_{k}+1, \lambda_{t}, \lambda_{k} \mid 1 \leqslant t \leqslant p\right.$ and $\left.Q_{t}(x, y) \neq 0\right\}$. By (44) and (6) we then obtain

$$
\operatorname{deg}\left(D(x) P_{n}(x)\right)=\operatorname{deg}\left(Q_{k}(x, n) x^{\nu_{k} n}\right) \text { for all } n>N .
$$

Hence $\operatorname{deg} P_{n}(x)=\nu_{k} n+d_{k}-\operatorname{deg} D(x)$ for all $n>N$.

\section{PRoOf of THE MAIN THEOREM}

In order to apply the result of the previous section we first recall a relationship between the Hilbert-Poincaré series and the regularity index. Let $A=A_{0}\left[\theta_{1}, \ldots, \theta_{r}\right]$, $\operatorname{deg}\left(\theta_{i}\right)=1$ for all $i=1, \ldots, r$. Let $M$ be a $\mathbb{Z}$-graded $A$-module. For an integer $j, M(j)$ denotes the $\mathbb{Z}$-graded $A$-module with the grading given by $M(j)_{a}=M_{a+j}$ for all $a \in \mathbb{Z}$. It is obvious that $\operatorname{ri}\left(I^{n} M(j)\right)=\operatorname{ri}\left(I^{n} M\right)-j$ for all $n \geqslant 0$. Shifting $M$ by a suitable integer $j$, we may assume that $M$ is positively graded, i.e., $M_{a}=0$ for all $a<0$. It is obvious that all modules $I^{n} M$ are also positively graded. Then, by the Hilbert-Serre theorem, the Hilbert-Poincaré series of $M$ can be written as

$$
H P_{M}(x):=\sum_{a=0}^{\infty} \ell_{A_{0}}\left(M_{a}\right) x^{a}=\frac{P(x)}{(1-x)^{r}}, \text { for some } P(x) \in \mathbb{Z}[x] .
$$

If $M \neq 0$, we have $\operatorname{deg} P(x) \geqslant 0$.

Lemma 2. If $M \neq 0$, then $\operatorname{ri}(M)=\operatorname{deg} P(x)-r+1$.

Proof. See [1, Proposition 4.1.12] or [5, Theorem 1.1 and Proposition 1.2].

Now we can prove the main theorem as follows. In order to study the behaviour of $\operatorname{ri}\left(I^{n} M\right)$ we use a bigraded structure on the Rees algebra $\mathfrak{R}(I)=\bigoplus_{n \geqslant 0} I^{n}$ defined by $\mathfrak{R}(I)_{(a, n)}=\left[I^{n}\right]_{a}$. The Rees module $\mathfrak{R}(I, M)=\bigoplus_{n \geqslant 0} I^{n} M$ is a finitely generated bigraded $\mathfrak{R}(I)$-module with $\mathfrak{R}(I, M)_{(a, n)}=\left[I^{n} M\right]_{a}$. Assume that $I$ is generated by homogeneous polynomials $f_{1}, \ldots, f_{s}$ with $d_{1}:=\operatorname{deg} f_{1}, d_{2}:=$ $\operatorname{deg} f_{2}, \ldots, d_{s}:=\operatorname{deg} f_{s}$. Then $\mathfrak{R}(I)=A_{0}\left[\theta_{1}, \ldots, \theta_{r}, f_{1} t, \ldots, f_{s} t\right]$ is a finitely generated bigraded algebra over the Artinian ring $A_{0}$ with $\operatorname{deg} \theta_{i}=(1,0)$, for all $i=1, \ldots, r$ and $\operatorname{deg} f_{j} t=\left(d_{j}, 1\right)$ for all $j=1, \ldots, s$. Since $M$ is a positively graded $A$-module, the Rees module $\mathfrak{R}(I, M)$ is a positively bigraded $\mathfrak{R}(I)$-module, i.e., $\mathfrak{R}(I, M)_{(a, n)}=0$ for all $a<0$ or $n<0$. The multi-graded version of the Hilbert-Serre theorem (see [5, Theorem 2.3]) says that the Hilbert-Poincaré series of the $\mathfrak{R}(I)$-module $\mathfrak{R}(I, M)$ can be written as

$$
\begin{aligned}
H P_{\mathfrak{R}(I, M)}(x, y) & :=\sum_{a, n \geqslant 0} \ell_{A_{0}}\left(\mathfrak{R}(I, M)_{(a, n)}\right) x^{a} y^{n}=\sum_{a, n \geqslant 0} \ell_{A_{0}}\left(\left[I^{n} M\right]_{a}\right) x^{a} y^{n} \\
& =\frac{P(x, y)}{(1-x)^{r}\left(1-y x^{d_{1}}\right) \cdots\left(1-y x^{d_{s}}\right)},
\end{aligned}
$$


where $P(x, y) \in \mathbb{Z}[x, y]$. For each $n \geqslant 0$, by (17), there is a polynomial $P_{n}(x) \in \mathbb{Z}[x]$ such that

$$
H P_{I^{n} M}(x)=\sum_{a=0}^{\infty} \ell_{A_{0}}\left(\left[I^{n} M\right]_{a}\right) x^{a}=\frac{P_{n}(x)}{(1-x)^{r}} .
$$

Together with (8), we then have the identity

$$
\sum_{n=0}^{\infty} P_{n}(x) y^{n}=\frac{P(x, y)}{\left(1-y x^{d_{1}}\right) \cdots\left(1-y x^{d_{s}}\right)} .
$$

Note that $P(x, y)$ is divisible by $\left(1-y x^{d_{1}}\right) \cdots\left(1-y x^{d_{s}}\right)$ in the $\operatorname{ring} \mathbb{Q}[x, y]$ if and only if $P_{n}(x)=0$ for all $n \gg 0$. But then $I^{n} M=0$ for all $n \gg 0$. Hence by the assumption, $P(x, y)$ is not divisible by $\left(1-y x^{d_{1}}\right) \cdots\left(1-y x^{d_{s}}\right)$. By (10) and Theorem 1, $\operatorname{deg} P_{n}(x)$ is a linear function of $n$ for all $n \gg 0$. The leading coefficient of this function is $d_{i}$ for some $i \in\{1, \ldots, s\}$. By (9) and Lemma 2, $r i\left(I^{n} M\right)=\operatorname{deg} P_{n}(x)-r+1$. Hence $\operatorname{ri}\left(I^{n} M\right)$ is a linear function of $n$ with the leading coefficient $d_{i}$ for all $n \gg 0$.

The following consequence answers the question posed in 3 . on the asymptotic behaviour of the function $\operatorname{ri}\left(I^{n}\right)$ for a homogeneous ideal $I$ in a polynomial ring.

Corollary 3. Let $A=K\left[X_{1}, \ldots, X_{r}\right]$ be a polynomial ring over a field $K$ and $I$ a non-zero proper homogeneous ideal of $A$. Let $f_{1}, \ldots, f_{s}$ be a minimal homogeneous basis of $I$ and $d_{i}=\operatorname{deg} f_{i}$ for all $i=1, \ldots, s$. Then we have

a) $\operatorname{ri}\left(I^{n}\right)$ is a linear function of $n$ for all $n \gg 0$.

b) $\operatorname{ri}\left(\overline{I^{n}}\right)$ is a linear function of $n$ for all $n \gg 0$, where $\overline{I^{n}}$ is the integral closure of $I^{n}$.

In each case, the leading coefficient of the corresponding linear function is one of the numbers $d_{1}, \ldots, d_{s}$.

Proof. The statement $(a)$ is the main theorem in the case $M=A$. The statement (b) follows from the fact that $\overline{I^{n}}=I^{n-n_{0}} \overline{I^{n_{0}}}$ for some $n_{0} \geqslant 0$ and all $n \geqslant n_{0}$.

Remark. Let $A=K\left[x_{1}, \ldots, x_{r}\right]$ be a polynomial ring over a field $K$. Let $M$ be a finitely generated graded $R$-module. Assume that $M$ has a minimal graded free resolution:

$$
0 \longrightarrow F_{p} \longrightarrow \cdots \longrightarrow F_{1} \longrightarrow F_{0} \longrightarrow M \longrightarrow 0 \text {. }
$$

Let $b_{i}(M)$ denote the maximal degree of the generators of $F_{i}$. The CastelnuovoMumford regularity of $M$ is defined by

$$
\operatorname{reg}(M)=\max \left\{b_{i}(M)-i \mid i=0, \ldots, p\right\} .
$$

If $I$ is a non-zero proper homogeneous ideal of $A$, then $\operatorname{reg}\left(I^{n}\right)=p(I) n+b$ for all $n \gg 0$, where $p(I)$ is a certain well-defined generating degree of $I$ (see [2] and [4]) and $b \in \mathbb{N}$. Let $r i\left(I^{n}\right)=d(I) n+c$ for all $n \gg 0$. Since $\operatorname{ri}\left(I^{n}\right) \leqslant \operatorname{reg}\left(I^{n}\right)$, we always have $d(I) \leqslant p(I)$. Of course, the equality occurs if the ideal $I$ is generated by elements of the same degree. In general, $d(I)$ may be arbitrarily less than $p(I)$.

Example ([3, Example 6]). Let $r, s \geqslant 1$, and

$$
I=\left(x_{1} x_{3}, x_{2} x_{3}, x_{1} x_{2} x_{4}\right)^{r}\left(x_{5}^{2}, x_{5} x_{6}^{2}\right)^{s} \subset A=K\left[x_{1}, x_{2}, x_{3}, x_{4}, x_{5}, x_{6}\right] .
$$

This ideal is generated in $r+s+1$ degrees: $2(r+s), \ldots, 2(r+s)+s, \ldots, 3(r+s)$. One can show that $\operatorname{ri}\left(I^{n}\right)=(2 r+3 s) n-1$ for all $n \geqslant 1$, while there is $b \in \mathbb{N}$ such that $\operatorname{reg}\left(I^{n}\right)=3(r+s) n+b$ for all $n \gg 0$. 


\section{ACKNOWLEDGMENTS}

I would like to thank my advisor, Le Tuan Hoa, for his advice and encouragement. The author was supported by the Vietnam National Science Foundation for Basic Research and the ICTP (Italy).

\section{REFERENCES}

[1] W. Bruns and J. Herzog, Cohen-Macaulay Rings, Cambridge University Press, 1993. MR:1251956 (95h:13020)

[2] S. D. Cutkosky, J. Herzog and N. V. Trung, Asymptotic behaviour of the CastelnuovoMumford regularity, Compositio Math. 118 (3) (1999), 243-261. MR1711319 (2000f:13037)

[3] L. T. Hoa and E. Hyry, On local cohomology and Hilbert function of powers of ideals, Manuscripta Math. 112, 77-92 (2003). MR2005931(2004i:13020)

[4] V. Kodiyalam, Asymptotic behaviour of Castelnuovo-Mumford regularity, Proc. Amer. Math. Soc. 128 (2) (2000), 407-411. MR.1621961 (2000c:13027)

[5] R. P. Stanley, Combinatorics and Commutative Algebra, second edition, Birkhäuser, Boston, MA, 1996. MR:1453579 (98h:05001)

[6] N. V. Trung and H.-J. Wang, On the asymptotic linearity of Castelnuovo-Mumford regularity, J. Pure Appl. Algebra 201 (2005), no. 1-3, 42-48. MR2158746 (2006k:13039)

Institute of Mathematics, 18 Hoang Quoc Viet Road, 10307 Hanoi, Viet Nam

E-mail address: tntrung@math.ac.vn 